\title{
Effects of solvent model flexibility on aqueous electrolyte behavior between electrodes
}

Richard L. Rowley

rowley@byu.edu

John N. Harb

john_harb@byu.edu

Clint G. Guymon

Matthew L. Hunsaker

Douglas Henderson

Follow this and additional works at: https://scholarsarchive.byu.edu/facpub

Part of the Chemical Engineering Commons

\section{Original Publication Citation}

C. G. Guymon, M. L. Hunsaker, J. N. Harb, D. Henderson, and R. L. Rowley, "Effects of solvent model flexibility on aqueous electrolyte behavior between electrodes", J. Chem. Phys. 118, $1195-122(23)$

\section{BYU ScholarsArchive Citation}

Rowley, Richard L.; Harb, John N.; Guymon, Clint G.; Hunsaker, Matthew L.; and Henderson, Douglas, "Effects of solvent model flexibility on aqueous electrolyte behavior between electrodes" (2003). Faculty Publications. 491.

https://scholarsarchive.byu.edu/facpub/491

This Peer-Reviewed Article is brought to you for free and open access by BYU ScholarsArchive. It has been accepted for inclusion in Faculty Publications by an authorized administrator of BYU ScholarsArchive. For more information, please contact ellen_amatangelo@byu.edu. 


\title{
Effects of solvent model flexibility on aqueous electrolyte behavior between electrodes
}

\author{
Clint G. Guymon, Matthew L. Hunsaker, John N. Harb, Douglas Henderson, ${ }^{\text {a) }}$ \\ and Richard L. Rowley ${ }^{\text {b) }}$ \\ Department of Chemical Engineering, Brigham Young University, Provo, Utah 84602-4100
}

(Received 26 December 2002; accepted 10 March 2003)

\begin{abstract}
Molecular dynamics simulations have been carried out for aqueous electrolyte solutions between model electrode surfaces. The effect of solvent model flexibility on bulk and double layer properties was observed for electrode surface charge densities of $0, \pm 0.1$, and $\pm 0.2 \mathrm{C} / \mathrm{m}^{2}$ and ion concentrations of $0,0.5$, and $1 \mathrm{M}$. Two flexible models were used to isolate the effects of flexibility from the effects of a change in the condensed-phase dipole moment. Model flexibility increases the pure water self-diffusion coefficient while a larger liquid dipole moment substantially decreases it. There is an increase in ion contact adsorption and counter ion affinity with the flexible models, suggesting that the ions are less tightly solvated. This conclusion is consistent with observed enhancements of solvated ion densities near uncharged electrodes for the flexible water case. Mobile ions in high concentration quickly damp out the electric field even at high electrode charge densities, but for dilute ion concentrations the field may extend to the center of the cell or beyond. In these cases it is more appropriate to integrate Poisson's equation from the electrode surface outward instead of the common method of assuming zero field at the center of the simulation cell. Using this methodology, we determine the voltage drop across the half-cell for both the rigid and flexible models. The half-cell voltage drop shows some dependence on ion concentration, but solvent flexibility has little effect on that behavior. (C) 2003 American Institute of Physics.
\end{abstract}

[DOI: $10.1063 / 1.1571056]$

\section{INTRODUCTION}

The study of interfacial properties using molecular simulations is becoming more widespread as computational capabilities increase and more accurate descriptions of intermolecular interaction potentials are developed. Molecular simulations show increasing promise in helping to understand interfacial/electrode behavior at a scale and for conditions that are prohibitive for other probing techniques. ${ }^{1-7}$ Simulations have also enhanced understanding of fluid structure near the electrode surface and provided important results for testing, improving, and extending theory. Recently, we performed molecular dynamics simulations on a model aqueous electrolyte solution between two electrodes ${ }^{1}$ using a rigid, nonpolarizable model for water. In that study, the potential in the interfacial region exhibited an oscillatory behavior near the electrode owing to the discrete nature of the molecular system. The simulated oscillations in the potential cannot be predicted from theories that do not include such a molecular description, but they are in qualitative agreement with the interfacial fluid structure suggested by simulation ${ }^{6}$ and experimental ${ }^{8}$ studies of a mercury-water interface.

Several recent studies ${ }^{9-14}$ of bulk water and electrolyte systems have used more sophisticated models that include polarization of the solvent molecules, self-consistent with the instantaneous molecular environment. Molecular polarizabil-

\footnotetext{
a) Department of Chemistry, Brigham Young University.

${ }^{b}$ Author to whom all correspondence should be addressed. Electronic mail: rowley@byu.edu
}

ity may impact the structure of the interfacial region near a charged surface and affect the solvation energy of the ions in the bulk. With this in mind, we have performed molecular dynamics (MD) simulations on aqueous electrolyte solutions confined between electrodes of varying applied charge using a flexible model for water that allows angle bending and bond vibrations. The resultant interfacial region structure and ion solvation characteristics are compared to our previous results for rigid water molecules with fixed dipole moments. This comparison allows us to focus on solvent effects resulting directly from the flexibility of the solvent and the concomitant polarizability, or change in dipole moment, created by this flexibility.

We also report the calculated electric field within the confined aqueous electrolyte obtained by integrating the onedimensional Poisson equation. Commonly the integration is performed from the center of the simulation cell, where the potential and field are assumed to vanish, to the electrode surface, but we find that for very dilute concentrations the electric field damps out asymptotically from the electrode surface and extends into the solution a long distance relative to the simulation cell length. This electric field penetration distance depends strongly on the concentration of mobile charge carriers in solution and, at least relative to the size of the simulation cell, approaches infinity in the limit of infinite dilution. Consequently, we have chosen to calculate the field by integrating Poisson's equation from the electrode surface outward by assuming a uniformly charged-sheet value for the field at the electrode surface. While this assumption is not 
TABLE I. Molecular models used in this study.

\begin{tabular}{llccc}
\hline \hline & & SPC/E & SPC/E-F & SPC/E-Fd \\
\hline$r_{\mathrm{OH}}^{\mathrm{o}}(\mathrm{nm})$ & Harmonic equilibrium OH bond distance & $0.100^{\mathrm{a}}$ & 0.100 & 0.099 \\
$<_{\mathrm{HOH}}^{\mathrm{o}}(\mathrm{rad})$ & Harmonic equilibrium HOH bond angle & $1.911^{\mathrm{a}}$ & 1.911 & 1.978 \\
$r_{\mathrm{OH}}(\mathrm{nm})$ & Average OH bond distance & $0.101(0.001)$ & $0.102(0.006)$ & $0.101(0.004)$ \\
$<_{\mathrm{HOH}}(\mathrm{rad})$ & Average HOH bond angle & $1.88(0.03)$ & $1.82(0.17)$ & $1.90(0.16)$ \\
$k_{b}\left(\mathrm{~kJ} / \mathrm{mol} / \mathrm{nm}^{2}\right)$ & Harmonic bond constant & $\ldots$ & $4.638 \times 10^{5}$ & $4.638 \times 10^{5}$ \\
$k_{a}\left(\mathrm{~kJ} / \mathrm{mol}^{\mathrm{b}} / \mathrm{rad}^{2}\right)$ & Harmonic angle constant & $\ldots$ & 384 & 384 \\
Freq $\left(\mathrm{cm}^{-1}\right)$ & Vibration frequencies & $\cdots$ & $1621,3662,3749$ & $1621,3663,3736$ \\
\hline
\end{tabular}

${ }^{a}$ Reference 15 .

${ }^{\mathrm{b}}$ Reference 17.

rigorous very near an electrode consisting of discretely charged molecules, we show that deviations from a threedimensional solution are small. Using this procedure, we are able to show the influence of ion concentration on the electric field and assess any limitations due to the computational domain.

\section{SIMULATION SPECIFICS}

We have used three different water models in our simulations. In our previous studies, $\mathrm{SPC} / \mathrm{E}^{15}$ water molecules were simulated between two model electrodes. ${ }^{1,2}$ In the SPC/E model, the dispersion interactions are treated with a spherical Lennard-Jones (LJ) potential located at the center of the oxygen atom. Polar interactions are included through equivalent partial positive charges located at the hydrogen nuclei, but the hydrogen atoms in this model do not have any LJ potentials assigned to them. An equivalent negative charge is also assigned to the oxygen atomic center. To examine the effects of polarization due to model flexibility, without a full relaxation of the electron density distribution, we have formulated a flexible SPC/E model, or SPC/E-F, in which a harmonic potential, ${ }^{16,17}$ coupled with a multipletime-scale integrator, is used to determine intramolecular motion. The equilibrium bond distances and bond angles for the isolated SPC/E-F molecule are identical to those in the SPC/E model. However, they are different for condensedphase simulations. To separate out flexibility effects from induced dipole effects caused by changes in the equilibrium geometry in the condensed phase, we performed simulations using a third model, SPC/E-Fd, in which the equilibrium bonds and angles in the liquid are fixed to produce the same dipole moment at our simulation conditions as the rigid SPC/E model. The bond and angle harmonic potential parameters for the flexible models are given in Table I.
As in our previous work, ${ }^{1,2}$ the model fluids were placed between two electrodes comprised of fixed charged LJ spheres. Simulations were performed at electrode charge densities of $0, \pm 0.1$, and $\pm 0.2 \mathrm{C} / \mathrm{m}^{2}$ and ion concentrations of $0,0.5$, and $1 \mathrm{M}$. The SPC/E-Fd model was used in simulations at $\pm 0.1 \mathrm{C} / \mathrm{m}^{2}$ electrode charge density and $1 \mathrm{M}$ ion concentration. The univalent ions were modeled as $\mathrm{LJ}$ spheres with mass, $\sigma$, and $\varepsilon / k$ values of $18.015,78.178 \mathrm{~K}$, and $0.3169 \mathrm{~nm}$, respectively, where $\sigma$ and $\varepsilon$ are the LJ size and energy parameters and $k$ is Boltzmann's constant. As in our previous work with a rigid solvent, the cation and anion are identical except for charge. The lengths of the cell in the $x, y$, and $z$ directions were $8.512,2.55$, and $2.55 \mathrm{~nm}$, respectively. The electrode surfaces were positioned at 0 and 4.256 $\mathrm{nm}$ so that half the cell was filled with fluid; the other half of the cell was empty space to damp out long-range interactions (see Ref. 18). Each electrode was modeled with 289 LJ atoms distributed as a single layer of a bcc lattice, exposing the $\left[\begin{array}{lll}1 & 0 & 0\end{array}\right]$ surface, having a lattice constant of $0.15 \mathrm{~nm}$. The LJ parameters for the electrode atoms were $\sigma=0.15 \mathrm{~nm}$ and $\varepsilon / k=50 \mathrm{~K}$. Lorentz-Berthelot combining rules were used to approximate the cross fluid-fluid and fluid-solid interactions between sites. Pairwise additivity of forces was also assumed.

Ten simulations were performed at each condition. All simulations were initiated from unique starting configurations and included 105 ps of equilibration and 200 ps of data collection. Results are an average of the ten runs performed at each condition. Coulomb real-space and LJ interactions were cut off at $1.0 \mathrm{~nm}$. Long-range Coulombic interactions were treated with the particle-particle-particle-mesh $\left(\mathrm{P}^{3} \mathrm{M}\right)$ technique, ${ }^{19}$ using an alpha value of $3.007 \mathrm{~nm}^{-1}$ and the Yeh and Berkowitz correction term. ${ }^{18}$ The $\mathrm{P}^{3} \mathrm{M}$ mesh size was $64 \times 16 \times 16$ in the $x, y$, and $z$ directions, respectively. The

TABLE II. Comparison of bulk properties obtained from the models used in this study to values obtained for three additional flexible models: Teleman-Jönsson-Engtrom (TJE)—Ref. 17, SPC/F-Ref. 16, and the modified Toukan-Rahman (SPC-mTR)-Ref. 25.

\begin{tabular}{lcccccc}
\hline \hline & SPC/E & SPC/E-F & SPC/E-Fd & TJE-F & SPC/F & SPC-mTR \\
\hline$T(\mathrm{~K})$ & 298 & 301 & 301 & 301 & 298 & 298 \\
$\rho\left(\mathrm{g} / \mathrm{cm}^{3}\right)$ & 1.08 & 1.08 & 1.08 & 1.00 & 0.996 & 0.998 \\
$U_{\text {inter }}(\mathrm{kJ} / \mathrm{mol})$ & $-48.6(0.3)$ & $-53.1(0.3)$ & $-48.0(0.3)$ & -45.3 & -41.2 & -48.71 \\
$U_{\text {intra }}(\mathrm{kJ} / \mathrm{mol})$ & $\cdots$ & $7.2(0.3)$ & $6.8(0.1)$ & 5.1 & 5.9 & 7.07 \\
$\langle\mu\rangle(\mathrm{D})$ & $2.41(0.06)$ & $2.54(0.31)$ & $2.39(0.27)$ & 2.44 & 2.30 & 2.44 \\
$D\left(10^{-5} \mathrm{~cm}^{2} / \mathrm{s}\right)$ & $2.18(0.01)$ & $1.75(0.01)$ & $2.63(0.01)$ & 6.1 & 4.6 & 2.70 \\
\hline \hline
\end{tabular}


(a)

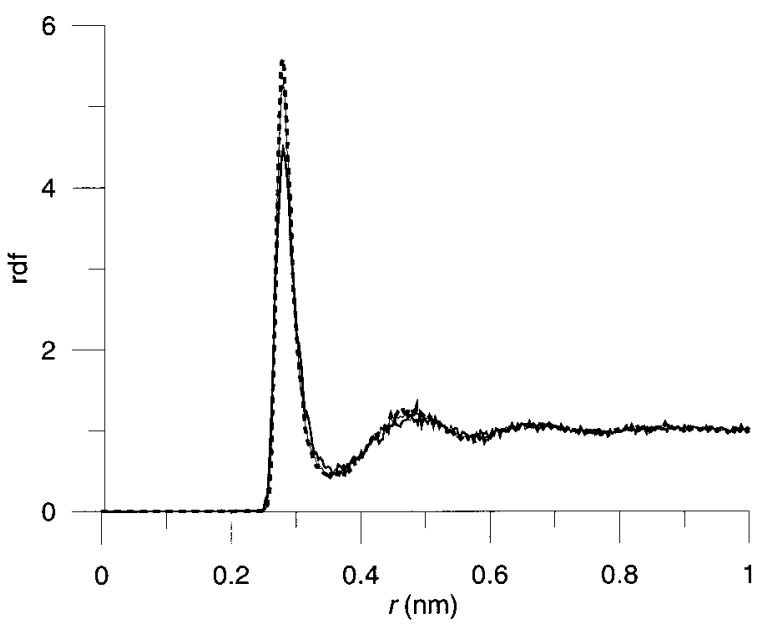

(b)

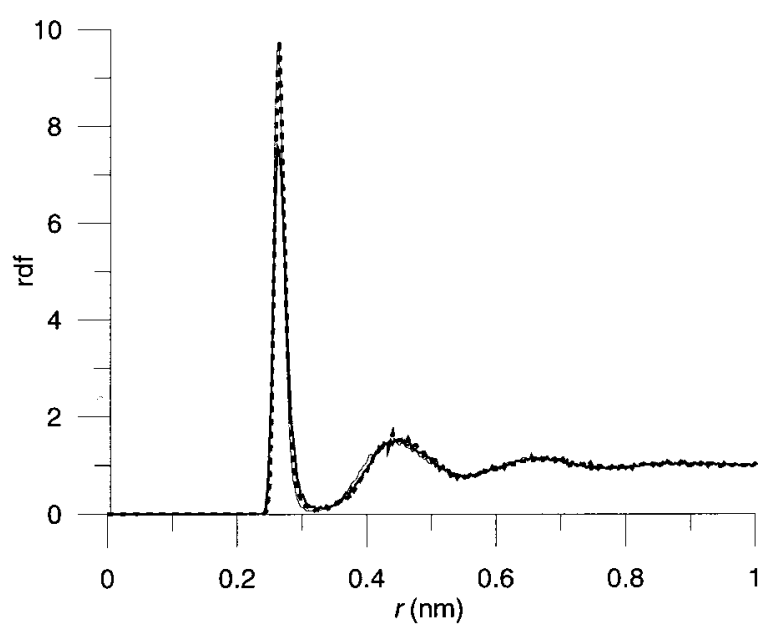

FIG. 1. Ion-O rdf for (a) cation and (b) anion using the SPC/E (-), SPC/ E-F (-), and SPC/E-Fd (---) models.

time step size was 2.5 fs. One-thousand mobile molecules were in each simulation at a bulk density of $60 \mathrm{~mol} / \mathrm{L}$ and a temperature of $300 \mathrm{~K}$. This density represents a relatively high pressure, but it satisfactorily avoids proximity to saturation where model inaccuracies could lead to the undesired presence of two phases. A fifth-order-correct predictorcorrector integrator was used in conjunction with Gaussian constraints for the rigid SPC/E model; an rRESPA algorithm $^{20,21}$ was used for the flexible models in which ten "fast" steps were integrated for each long step.

\section{RESULTS AND DISCUSSION}

\section{A. Differences in bulk properties between the three models}

To compare the differences between solvent models in bulk phase properties, bulk NVT simulations with periodic boundaries (no electrodes) were performed. In these cases, 1000 molecules were simulated in a cubic box of length $3.025 \mathrm{~nm}$. The results for the SPC/E-F model in Table I show that the introduction of flexibility increases the $\mathrm{OH}$ bond lengths and compresses the $\mathrm{HOH}$ angles. The combination results in a $5 \%$ increase in the condensed-phase dipole moment as shown in Table II. The potential energy of the sys- (a)

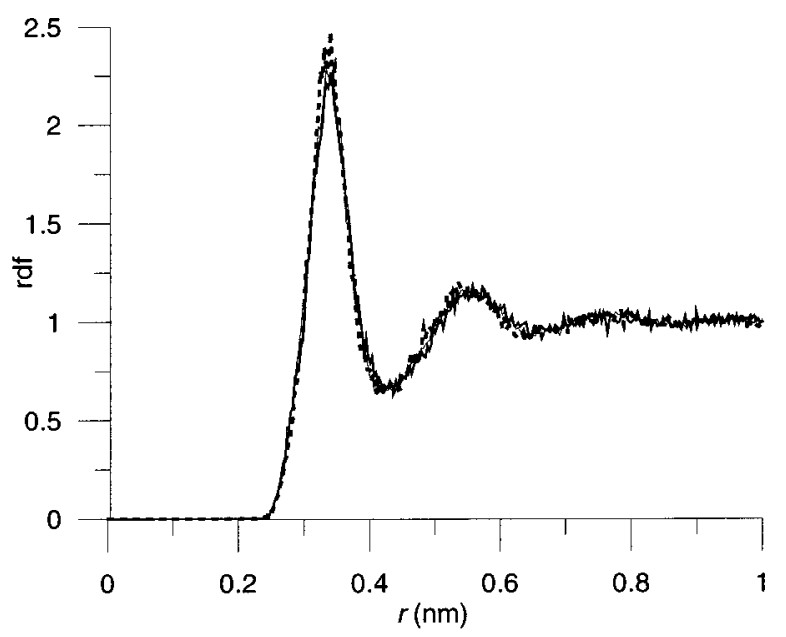

(b)

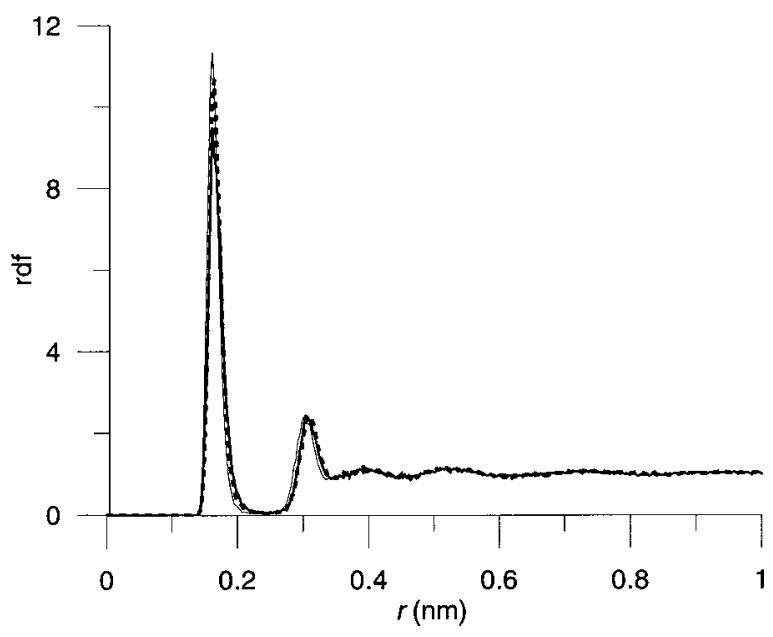

FIG. 2. Ion-H rdf for (a) cation and (b) anion using the SPC/E (一), SPC/ E-F (-), and SPC/E-Fd (---) models.

tem is lower for the flexible model because of the increased equilibrium dipole moment and resultant stronger hydrogen bonds. The self-diffusion coefficient is also decreased, presumably by this same mechanism. Results for the SPC/E-Fd model show that the decrease in potential energy for SPC/ $\mathrm{E}-\mathrm{F}$ is due to the increased dipole moment as the potential energy for SPC/E-Fd is nearly equal to that of the rigid model. Interestingly, flexibility by itself (SPC/E-Fd) increases the self-diffusion coefficient. This effect was also observed by Tironi et al. ${ }^{16}$ and Teleman and Jönsson. ${ }^{17}$ The increase in the self-diffusion coefficient due solely to flexibility may be due to increased mobility and reduced drag as molecules can deform to move past one another. This explanation is consistent with results from other simulations that indicate that flexibility decreases viscosity. ${ }^{22,23}$ Table II also compares bulk properties (intermolecular potential energy, $U_{\text {inter }}$, intramolecular potential energy, $U_{\text {intra }}$, average dipole moment $\langle\mu\rangle$, and self-diffusion coefficient, $D$ ) obtained using the models used in this study with values reported for other flexible models already in the literature. The frequencies of condensed phase bending and symmetric/asymmetric stretch of the flexible models agree well with water vapor experimental values ${ }^{24}\left(1595,3657\right.$, and $\left.3756 \mathrm{~cm}^{-1}\right)$. It is known 
(a)

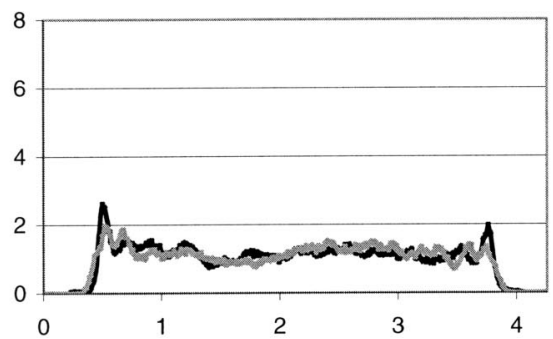

(b)

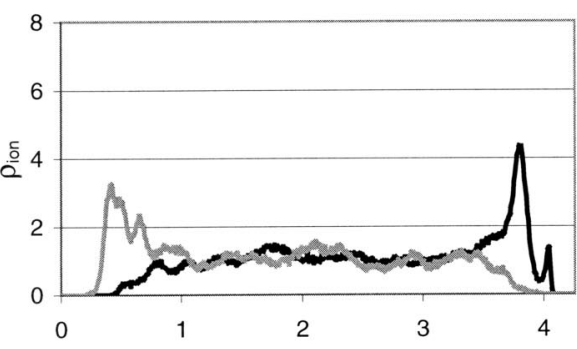

(c)

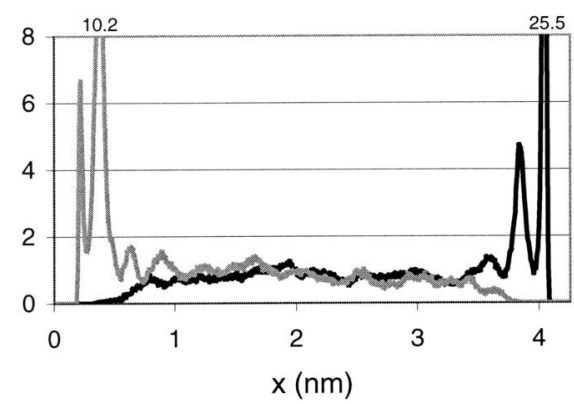

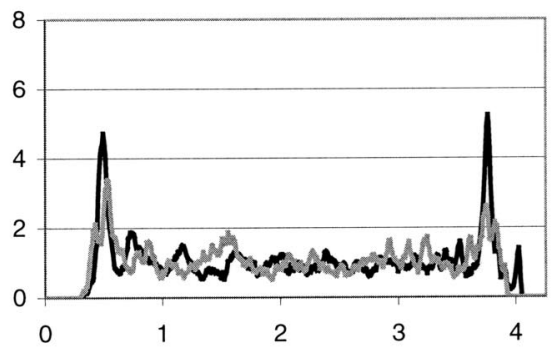

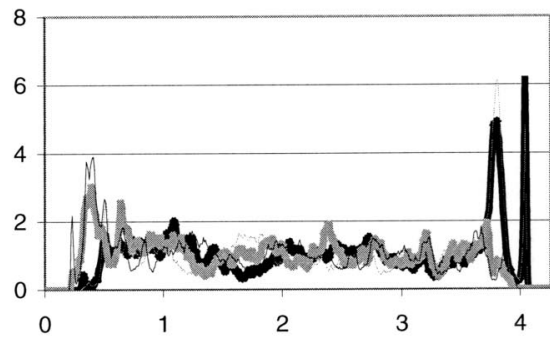

FIG. 3. Cation (一, black) and anion (-, gray) average ion density profiles as a function of distance away from the positively charged electrode for 1 $\mathrm{M}$ bulk concentration. The negative electrode is located at $4.256 \mathrm{~nm}$. Electrode charge densities are (a) 0.0, (b) \pm 0.1 , and (c) $\pm 0.2 \mathrm{C} / \mathrm{m}^{2}$. SPC/E model results are on the left, SPC/E-F on the right. In the case of $\pm 0.1 \mathrm{C} / \mathrm{m}^{2}$ the SPC/E-Fd model cation (-, thin) and anion $(---$, thin) results are overlaid. that the IR absorbance for these same stretching modes decreases in the condensed-phase water; no density dependence is observed here owing to the use of harmonic intramolecular potentials. $^{25}$

\section{B. Ion hydration results in the absence of electrodes}

A comparison of bulk solution structure characteristic of the models was investigated by computing the ion-oxygen radial distribution function (rdf). Figure 1(a) shows a comparison of the cation-O rdf obtained for the various models using an average of five independent runs at an ion concentration of $0.063 \mathrm{M}$. Ion centers were excluded from the rdf averages if an ion-ion distance was less than $2 \mathrm{~nm}$ so as to compare the ion-O rdf functions without possible ion pairing. Figure 1(b) shows a similar comparison for the anion-O rdf. Flexibility is seen to increase the first peak height for both ion models. While a similar effect is observed in Fig. 2(b) for the anion-H rdf, flexibility has little apparent effect on the cation-H rdf shown in Fig. 2(a). The increased dipole moment of the SPC/E-F model does produce a slight increase in the anion-H rdf with a minor shift to shorter distances in the second peak, but much of the increase in peak height seems to be due to the flexibility itself. These firstpeak results suggest that the primary effect of the flexible solvent is packing, i.e., slight deformation of the solvent molecules permitting higher density in the first coordination shell. Due to the asymmetry in the model water molecules, the negative ion is more strongly hydrated as seen by the location and magnitude of the first peak in the rdf plots. This is because in this study the anion and cation are of equal size and so the partial positive charge on the hydrogen sites can get much closer to the charge center of the counter-charged ion than can the partial negative charge at the center of the oxygen site. Obviously, in real systems where the anion is often significantly larger than the cation, this same chargeseparation explanation would suggest that the anion would be less strongly hydrated.

\section{Ion hydration in proximity to the electrode surface}

Figure 3 shows the ion density distributions obtained from the simulations for different charges on the electrodes at a bulk ion concentration of $1 \mathrm{M}$ (compare with Ref. 26). Results for the flexible model are shown at the right; results for the rigid model are shown on the left. The results for the $\mathrm{SPC} / \mathrm{E}-\mathrm{Fd}$ model at an electrode charge density of \pm 0.1 $\mathrm{C} / \mathrm{m}^{2}$ are overlaid on the SPC/E-F plot in Fig. 3(b) and are similar to those of the other flexible model. Close examination shows an additional anion peak close to the wall for the SPC/E-Fd model and increased height in other peaks near the wall indicating weaker hydration relative to the SPC/E-F model. However, the differences between the SPC/E-Fd and $\mathrm{SPC} / \mathrm{E}-\mathrm{F}$ results are small relative to the difference between 
(a)

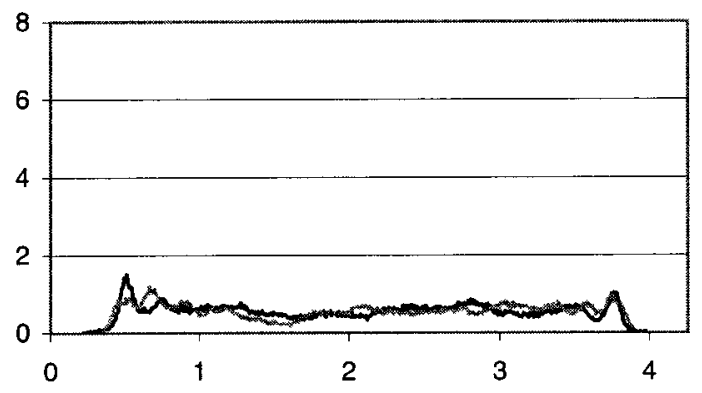

(b)
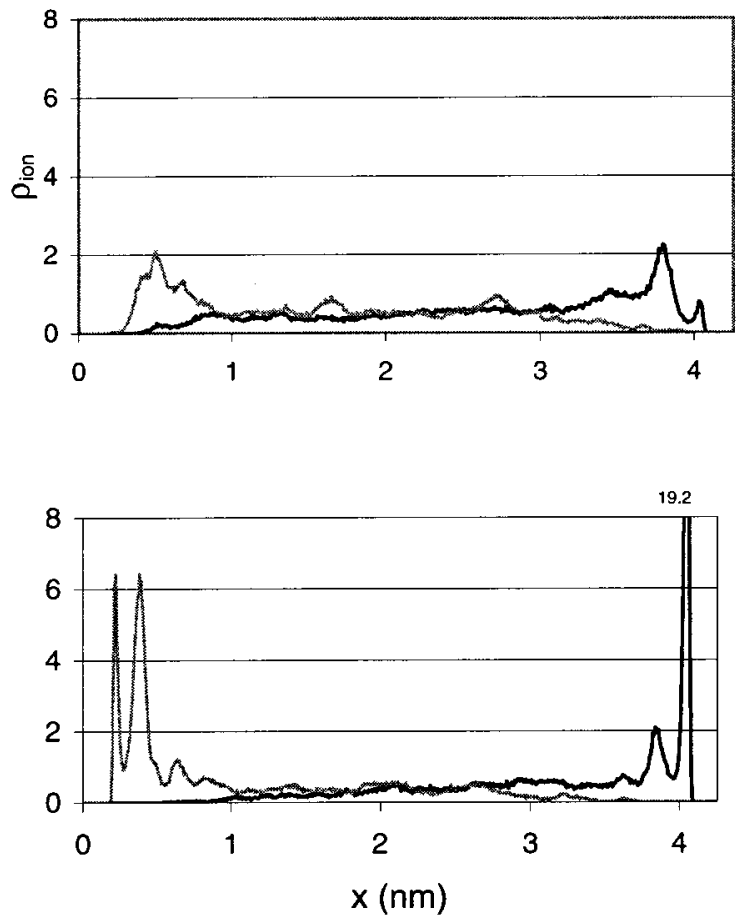
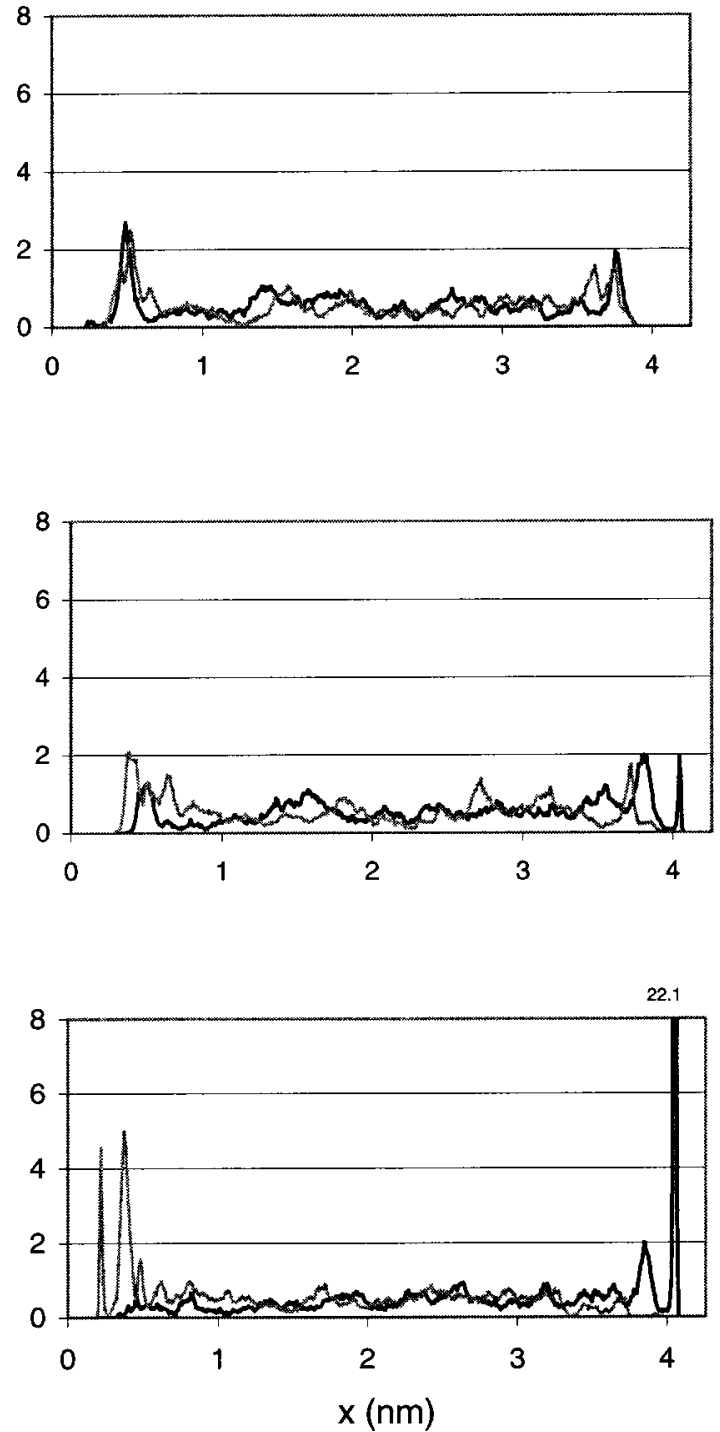

FIG. 4. Cation (-) and anion (-) average ion density profiles as a function of distance away from the positively charged electrode for $0.5 \mathrm{M}$ bulk concentration. The negative electrode is located at $4.256 \mathrm{~nm}$. Electrode charge densities are (a) 0.0, (b) \pm 0.1 , and (c) $\pm 0.2 \mathrm{C} / \mathrm{m}^{2}$. SPC/E model results are on the left, SPC/E-F on the right.

the results of either of these models and the rigid model. We therefore show comparisons only between the SPC/E and SPC/E-F models at the remaining conditions. Figure 3(a) shows this comparison for uncharged electrodes. The presence of the primarily repulsive wall terminates the hydrogenbonding network within the solvent, and water molecules have a preferred orientation at even an uncharged wall (compare Fig. 5). This orientation of the dipolar solvent creates a small field. As can be seen in the left-hand plot of Fig. 3(a) for SPC/E water, there is a small enhancement of the ion densities in the so-called outer-Helmholtz plane (OHP) of both electrodes due to this solvent orientation propensity. However, the more tightly hydrated ions with the SPC/E model do not permit as much orientation of the solvent at the wall in response to the termination of the hydrogen bonding as do the less-tightly bound ions hydrated with the SPC/E-F model. The flexible model permits higher solvent orientation at the walls in the presence of ions with a resultant larger ion density enhancement at both neutral electrodes as shown on the right-hand side of Fig. 3(a). Evidence of the weaker hy- dration of the ion with SPC/E-F can also be seen by the increased ion affinity in Fig. 3(a) (right), characterized by the close proximity of paired counter-ion peaks near the electrode surface not present for the rigid model. When the charge on the wall is increased to $\pm 0.1 \mathrm{C} / \mathrm{m}^{2}$, flexibility enhances the cation contact adsorption (the inner-Helmholtz plane or IHP) which is not as pronounced with the SPC/E model. Little effect is observed for the anion. Contact adsorption is again enhanced by solvent flexibility at a charge density of $\pm 0.2 \mathrm{C} / \mathrm{m}^{2}$ shown in Fig. 3(c). Contact adsorption of both ions is significantly enhanced by the solvent's flexibility. Again note the ion affinity at these higher charges for the flexible solvent. Figure 3(c) shows that, in comparison to the rigid model and even in comparison to the flexible model at $\pm 0.1 \mathrm{C} / \mathrm{m}^{2}$, some of the cations are pulled back toward the positive electrode and some of the anions are pulled closer to the negative electrode by their counter ions. The flexible ion density plots are also seen to be rougher than their rigid counterparts, though they represent averages over the same time period, suggesting that the system with the flexible- 
solvated ions tends to equilibrate more slowly. This decreased ion mobility may be due to an increase in ion-ion interactions and a stronger water hydrogen-bond network, both owing to the weaker ion-solvent interaction for the flexible solvent.

Results at a lower ion concentration, $0.5 \mathrm{M}$, are shown in Fig. 4. At this concentration, the effects of flexibility on the IHP are less prominent. However, ion affinity is again seen to be more dominant for the flexible solvent. Strong ion affinity is seen with electrode charge densities of \pm 0.1 and \pm 0.2 $\mathrm{C} / \mathrm{m}^{2}$.

The ion profiles that are plotted in Figs. 3 and 4 and, indeed, the water molecule profiles that we have not plotted, have appreciable maxima and minima (see also Refs. 1, 2, and 27). This is in pronounced contrast to the prediction of monotonic profiles by the venerable Gouy-Chapman theory that neglects the size of the ions and replaces the discrete water molecules by a continuum. Such nonmonotonic profiles have been predicted by theory ${ }^{28}$ for nearly three decades and have been seen in recent experiments.

\section{Electrical properties of the double layer}

The electric potential, $\phi$, at any point $x$ in the interfacial region may be calculated by integrating the one-dimensional Poisson equation from infinite distance from the electrode to $x,{ }^{28}$ or

$$
\phi(x)=-\frac{e}{\varepsilon_{0}} \sum_{i} z_{i} \int_{\infty}^{x}\left(x-x^{\prime}\right) \rho_{i}\left(x^{\prime}\right) d x^{\prime},
$$

where $\rho_{i}$ is the local density of site $i$ which has charge $z_{i}$, and $\varepsilon_{0}$ is the permittivity of free space. In previous studies $^{1,18}$ it has been assumed that the MD simulation cell size is large enough to apply Eq. (1) from the center of the cell to $x$. The validity of this assumption of zero field, $E$ $=-d \phi / d x=0$, at the center of the simulation cell depends upon the relative sizes of the MD cell and the double layer; the latter is a function of the electrode charge and the ion concentration. Because orientation of the water molecules constitutes a sensitive measure of the field in the cell, we have examined the zero-field assumption in terms of the water dipole distribution in Fig. 5. Water orientation in Fig. 5 is characterized by the projection of the water dipole onto the normal vector for each electrode, pointing into the cell, or $\cos \theta$. Shown are the distributions of $\cos \theta$ for the rigid $\mathrm{SPC} / \mathrm{E}$ model as a function of cell position for pure water at electrode charge densities of 0 [Fig. $5(\mathrm{a})]$ and $\pm 0.1 \mathrm{C} / \mathrm{m}^{2}$ [Fig. 5(b)] and for a nominal concentration of $1 \mathrm{M}$ ions added with an electrode charge density of $\pm 0.1 \mathrm{C} / \mathrm{m}^{2}[$ Fig. $5(\mathrm{c})$ ]. Note that by referencing $\theta$ to each electrode, there is a sign reversal at the center of the simulation cell for $\cos \theta$ in Fig. 5. In Fig. 5(a), a preferred orientation of water molecules at the electrode walls produces a net dipole near the wall that damps out quickly with distance from the surface. Hence, the assumption of zero field at the midpoint of the cell is valid for the conditions shown in Fig. 5(a). However, in Fig. 5(b) the dipolar solvent cannot damp out the field because there are no mobile charges present. In Fig. 5(c), the addition of ions eliminates most of the field, but there is yet (a)

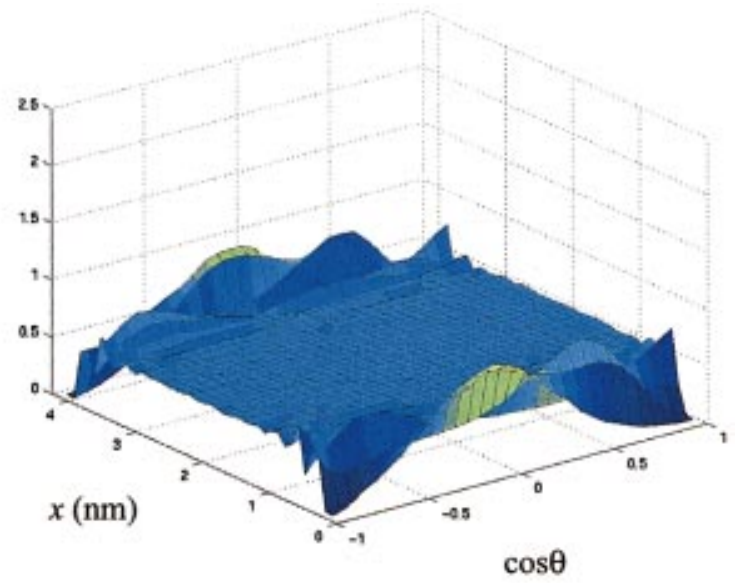

(b)

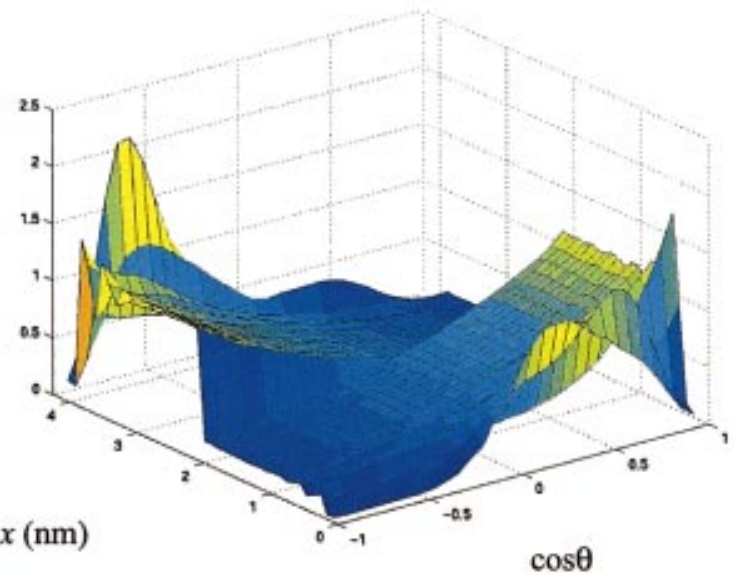

(c)

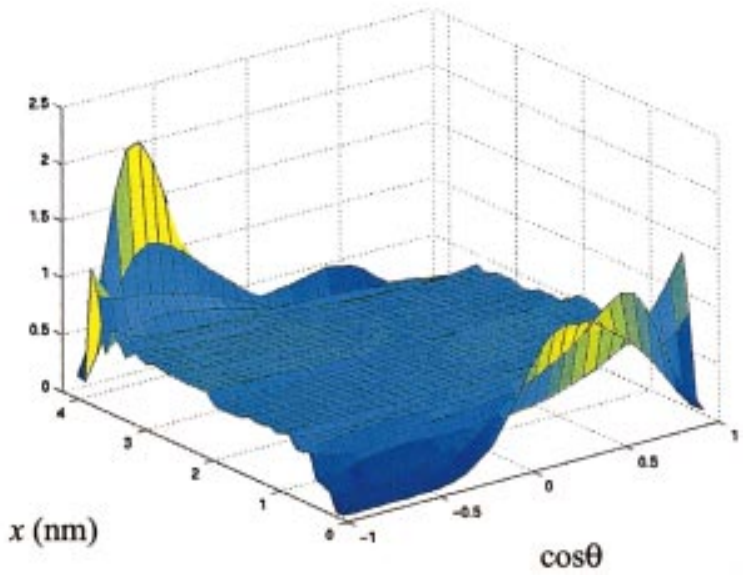

FIG. 5. (Color) Dipole orientation surfaces for SPC/E model water at an electrode charge density and ion concentration of (a) $0.0 \mathrm{C} / \mathrm{m}^{2}$ and $0 \mathrm{M}$, (b) $\pm 0.1 \mathrm{C} / \mathrm{m}^{2}$ and $0 \mathrm{M}$, and $(\mathrm{c}) \pm 0.1 \mathrm{C} / \mathrm{m}^{2}$ and $1 \mathrm{M}$. The positively charged electrode is located at 0 with the negative electrode at $4.256 \mathrm{~nm}$.

a small field at the center of the cell as evidenced by the slight bump in the profile. The diffuse layer in which there is still a small field present is quite large because of the decreasing diffusional response of the ions to the diminishing field further out from the electrode surface. The assumption of a zero field at the center of the cell may be problematic in this case. Polarizability in the SPC/E-F model enhances the dipole orientations at the electrode surface and shields the field to a greater degree. This also enhances the possibility of counter ion affinity as previously noted. 


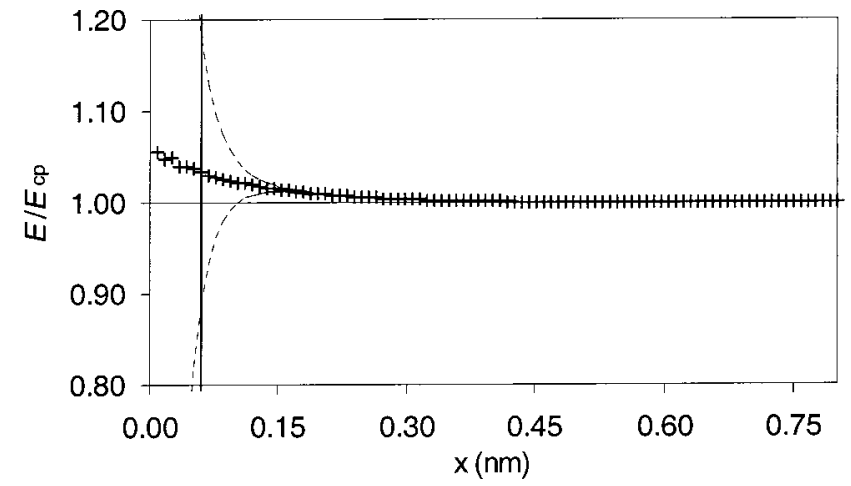

FIG. 6. Average electric field deviations $(+++)$ from the uniformly charged plate value as a function of distance from the bcc $\left[\begin{array}{lll}1 & 0 & 0\end{array}\right]$ pointcharged electrode surface with a lattice constant of $0.15 \mathrm{~nm}$. The dashed lines represent a one standard deviation range and the vertical line represents the distance of closest approach of any charge in our MD simulations.

As an alternative to finding the potential from Eq. (1), one can integrate Poisson's equation from the electrode surface to $x$, thereby avoiding the possibility of finite cell size problems. In so doing, the field at the electrode surface $(x$ $=0$ ) is obtained from the applied external field. In this work, we have assumed the field present at the electrode is equal to the uniformly charged sheet value, $q / \varepsilon_{0}$, to obtain

$$
\phi(x)=-\frac{e}{\varepsilon_{0}} \sum_{i} z_{i} \int_{0}^{x}\left(x-x^{\prime}\right) \rho_{i}\left(x^{\prime}\right) d x^{\prime}-\frac{q}{\varepsilon_{0}} x+\phi(0)
$$

for the potential distribution, where $q$ is the charge density at the electrode surface $(x=0)$, and $\phi(0)$ is a constant at the electrode surface.

Because our model electrodes are molecular in nature with a body-centered-cubic distribution of charges in the $y z$ plane, the one-dimensional Poisson equation with a charged sheet boundary condition is not rigorous very near the electrode surface. To test deviations of the field near our molecular electrode from that of a charged plate, test charges were used to probe the field (with no solution present) as a function of distance from the electrode. Figure 6 shows the average electric field divided by the charged plate value, $E_{\mathrm{CP}}$, obtained from the test charges using an Ewald sum with a convergence parameter of $2.87 \mathrm{~nm}^{-1}$. As can be seen from the average value and the one-sigma confidence interval plotted in Fig. 6, the approximation of replacing $E$ with $E_{\mathrm{CP}}$ at the electrode produces an average or systematic error of less than 3\% (though deviations from the homogeneous field assumption for individual ions can be larger) in the field at the distance of closest approach (as obtained from the simulations and shown by the vertical line in Fig. 6) of a charged site. The error introduced by this assumption is seen to be even smaller elsewhere in the double layer.

Figure 7 shows voltage versus cell position (for the SPC/E model with an electrode charge density of \pm 0.2 $\mathrm{C} / \mathrm{m}^{2}$ ) calculated from Eq. (2). The observed nonmonotonic behavior of the potential drop mirrors very well the results obtained by Philpott and $\mathrm{Glosli}^{29}$ and $\mathrm{Spohr}^{30}$ for $\mathrm{NaCl}$ solutions. It is also evident that increasing charge carrier concentration better shields the electric field produced by the

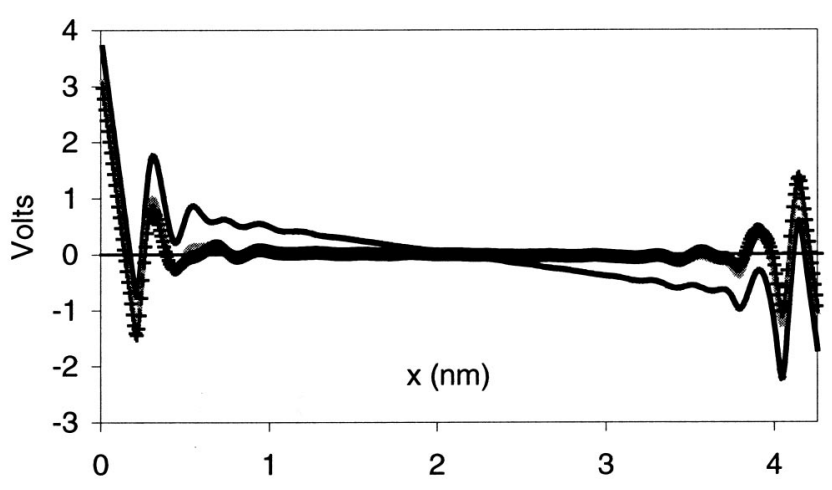

FIG. 7. Voltage drop profile for SPC/E water at an electrode charge density of $\pm 0.2 \mathrm{C} / \mathrm{m}^{2}$ with an ion concentration of $0(-), 0.5(-)$, and $1(++++)$ $\mathrm{M}$. Voltage set to zero at cell center.

charge density in the electrodes. Note that the constant in Eq. (2), $\phi(0)$, was fixed to give a value of zero for the voltage at the center of the simulation cell. The flexible counterpart of Fig. 7 possesses the same shape with slightly different halfcell voltage drops.

We note that the potential profiles of Figs. 6 and 7 reflect the nonmonotonic density profiles of the ions and water molecules. The potential profiles predicted by the GouyChapman theory are monotonic. Thus, the Gouy-Chapman profiles are qualitatively and quantitatively in error. Quantitative errors in the Gouy-Chapman potentials are usually overcome by semiempirical adjustment of parameters; however, no amount of parameter adjustment can overcome the observed qualitative errors.

Figure 8 shows the half-cell voltage drop versus the electrode charge density for the SPC/E-F model; results for $\mathrm{SPC} / \mathrm{E}$ are not shown but are visually indistinguishable. The voltage drop is approximately linear with charge density. It is of interest to note that the slope of this relationship is smaller for negative electrode charges. Likewise, the experimental curves are not symmetric. The voltage is a measure of the charge separation. Thus, the asymmetry in Fig. 8 agrees with

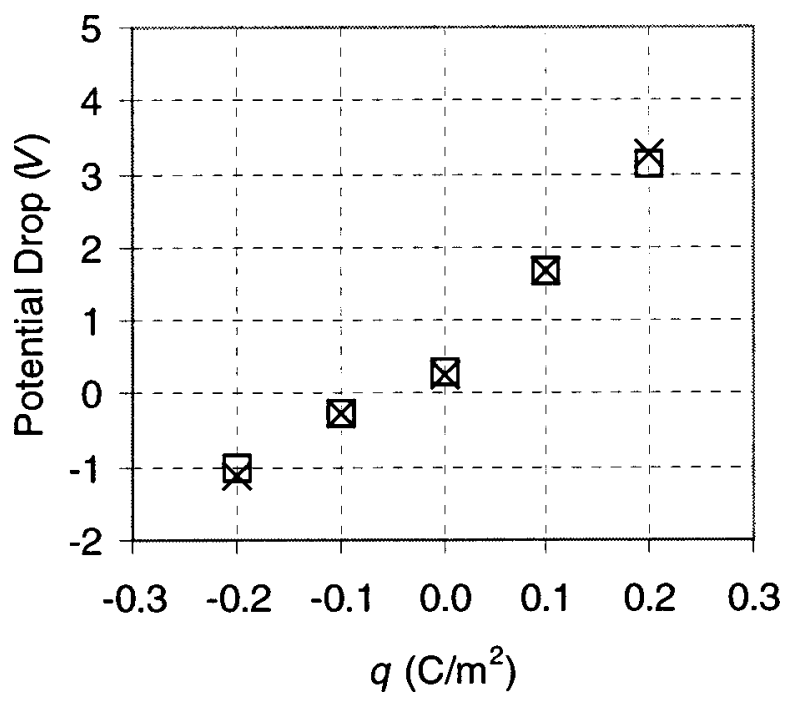

FIG. 8. Half-cell potential drop as a function of electrode charge for 0.5 $(\times)$, and $1 \mathrm{M}(\square)$ ion concentrations as obtained from the SPC/E-F model. 
TABLE III. Electric field (V/nm) and 95\% confidence interval (in parentheses) at the center of the simulation cell.

\begin{tabular}{lcrcc}
\hline \hline & & \multicolumn{3}{c}{$q\left(\mathrm{C} / \mathrm{m}^{2}\right)$} \\
\cline { 3 - 5 } Model & Ion conc. (M) & \multicolumn{1}{c}{0.0} & 0.1 & 0.2 \\
\hline SPC/E & 0.0 & $0.00(0.03)$ & $0.13(0.04)$ & $0.43(0.05)$ \\
& 0.5 & $0.00(0.07)$ & $0.03(0.14)$ & $0.06(0.09)$ \\
& 1.0 & $0.00(0.12)$ & $0.03(0.11)$ & $0.02(0.10)$ \\
SPC/E-F & 0.0 & $0.00(0.03)$ & $0.10(0.05)$ & $0.31(0.05)$ \\
& 0.5 & $-0.02(0.14)$ & $0.11(0.15)$ & $0.13(0.12)$ \\
& 1.0 & $0.00(0.11)$ & $0.03(0.16)$ & $0.10(0.10)$ \\
\hline \hline
\end{tabular}

the results in Figs. 3 and 4 where on average the cations are closer to the negatively charged electrode than are the anions to the positively charged electrode. The water molecules reinforce this effect. Geometrically, the positively charged hydrogen atoms in the water molecules can approach closer to the electrode than can the oxygen atoms. A smaller charge separation next to the negative electrode results in a smaller potential difference for a given magnitude of the electrode charge.

The asymmetry seen in Fig. 8 is a feature of the model parameters employed here, in particular the equal ion size for anions and cations. Generally, anions are larger in diameter and, more importantly, less fully hydrated than are cations. It is anticipated that this ion size effect, plus any asymmetry in the response of electrons of the electrode to the electrode charge, could easily shift the asymmetry so that it is the slope of the potential difference for positive electrode charge that is smaller as is seen in most experiments.

Values of the electric field calculated at the center of the simulation cell for the various conditions are shown in Table III. They suggest that the thickness of the interfacial region is increased by solvent flexibility. This result may be due to the decreased ability of the mobile charge carriers to shield the field because of the enhanced counter ion affinity.

\section{CONCLUSIONS}

Introducing flexibility into the SPC/E model causes significant changes in solvent properties. We have used two flexible models, SPC/E-F and SPC/E-Fd, to distinguish effects due to a flexible geometry from those that result from the changed dipole moment in the condensed phase. Both effects appear to impact the diffusion coefficient of the pure solvent while the model effect on total energy is due primarily to the dipole moment change. Both effects tend to increase the height of the first oxygen and hydrogen ion rdf peaks. The increase in rdf peak height appears to be mainly due to geometrical packing considerations as evidenced by detailed density profiles that suggest the hydration strength of the ions is smaller for the flexible solvent models. Ion density profiles show that contact adsorption at electrode surfaces is enhanced with solvent flexibility. Ion affinity is also enhanced when the ions are solvated with flexible water molecules.

We have also shown that care must be taken when using finite-sized cells with low concentrations of ions in a strong externally applied field. Although the mobile charges dampen out most of the field rather quickly, a small solvent dipole orientation may persist for a significant distance into the diffuse layer. In such cases, a good alternative to assuming an infinite cell length, or zero field at the center of the simulation cell, is integrating Poisson's equation from the electrode surface outward. We have shown that this can be done with good accuracy by using $E_{\mathrm{CP}}$ for the field at the electrode surface even when the electrode is modeled with fixed molecular centers and these discrete centers constitute the loci of any added electrode charges. Using this method for calculating the field, we found that the half-cell voltage drop is a function of the electrode charge and bulk ion concentration.

\section{ACKNOWLEDGMENTS}

This work was supported in part by a grant from the National Science Foundation (No. CTS-0215786) and by an undergraduate Mentoring Environment Grant (MEG) at Brigham Young University. We are appreciative of these grants and the super-computing facilities at Brigham Young University provided by Ira and Marylou Fulton.

${ }^{1}$ P. S. Crozier, R. L. Rowley, and D. Henderson, J. Chem. Phys. 113, 9202 (2000).

${ }^{2}$ P. S. Crozier, R. L. Rowley, and D. Henderson, J. Chem. Phys. 114, 7513 (2001).

${ }^{3}$ D. Michael and I. Benjamin, J. Electroanal. Chem. 450, 335 (1998).

${ }^{4}$ S.-J. Marrink, M. Berkowitz, and H. J. C. Berendsen, Langmuir 9, 3122 (1993).

${ }^{5}$ I.-C. Yeh and M. L. Berkowitz, Chem. Phys. Lett. 301, 81 (1991).

${ }^{6}$ E. Spohr, G. Toth, and K. Heinzinger, Electrochim. Acta 41, 2131 (1996).

${ }^{7}$ I.-C. Yeh and M. L. Berkowitz, J. Chem. Phys. 110, 7935 (1999).

${ }^{8}$ L. Bosio, R. Cortes, M. Denoziere, and G. Folcher, Coll. Phys. C7, Suppl. 10 50, 23 (1989).

${ }^{9}$ A. P. Lyubartsev, K. Laasonen, and A. Laaksonen, J. Chem. Phys. 114, 3120 (2001).

${ }^{10}$ G. Toth, J. Chem. Phys. 105, 5518 (1996).

${ }^{11}$ D. Laria and R. Fernandez-Prini, J. Chem. Phys. 102, 7664 (1995).

${ }^{12}$ E. Stockelmann, E. M. Aydt, and R. Hentschke, J. Mol. Model. [Electronic Publication] 3, 347 (1997).

${ }^{13}$ A. Kohlmeyer, W. Witschel, and E. Spohr, Chem. Phys. 213, 211 (1996).

${ }^{14}$ E. Oyen and R. Hentschke, Langmuir 18, 547 (2002).

${ }^{15}$ H. J. C. Berendsen, J. R. Grigera, and T. P. Straatsma, J. Phys. Chem. 91, 6269 (1987)

${ }^{16}$ I. G. Tironi, R. M. Brunne, and W. F. van Gunsteren, Chem. Phys. Lett. 250, 19 (1996).

${ }^{17}$ O. Teleman, B. Jönsson, and S. Engström, Mol. Phys. 60, 193 (1987).

${ }^{18}$ I.-C. Yeh and M. L. Berkowitz, J. Chem. Phys. 111, 3155 (1999).

${ }^{19}$ R. W. Hockney and J. W. Eastwood, Computer Simulation Using Particles (IOP, Bristol, 1988).

${ }^{20}$ S. J. Stuart, R. Zhou, and B. J. Berne, J. Chem. Phys. 105, 1426 (1996).

${ }^{21}$ M. Tuckerman, B. J. Berne, and G. J. Martyna, J. Chem. Phys. 97, 1990 (1992).

${ }^{22}$ N. G. Fuller and R. L. Rowley, Int. J. Thermophys. 21, 45 (2000).

${ }^{23}$ Y. Yang, Ph.D. dissertation, Brigham Young University, Provo, Utah, 2001.

${ }^{24}$ D. Eisenberg and W. Kanzman, The Structure and Properties of Water (Oxford University Press, London, 1969).

${ }^{25}$ C. C. Liew, H. Inomata, and K. Arai, Fluid Phase Equilib. 144, 287 (1998)

${ }^{26}$ E. Spohr, J. Electroanal. Chem. 450, 327 (1998).

${ }^{27}$ E. Spohr, in Solid-Liquid Electrochemical Interfaces, edited by G. Jerkiewicz, M. P. Soriaga, K. Uosaki, and A. Wieckowski [ACS Symp. Ser. 656, 31 (1997)].

${ }^{28}$ W. Schmickler and D. Henderson, Prog. Surf. Sci. 22, 323 (1986)

${ }^{29}$ M. R. Philpott, J. N. Glosli, in Solid-Liquid Electrochemical Interfaces, edited by G. Jerkiewicz, M. P. Soriaga, K. Uosaki, and A. Wieckowski [ACS Symp. Ser. 656, 13 (1997)].

${ }^{30}$ E. Spohr, Electrochim. Acta 44, 1697 (1999). 